\title{
Molecular sexing of African rhinoceros
}

\author{
Lindsay Peppin ${ }^{1}$, Ross McEwing ${ }^{2}$, Rob Ogden $^{3}$, Robert Hermes ${ }^{4}$, Cindy Harper ${ }^{5}$, \\ Alan Guthrie ${ }^{5}$ and Gary R. Carvalho ${ }^{1}$
}

(1) Molecular Ecology and Fisheries Genetics Laboratory, School of Biological Sciences, Environment Centre Wales, Bangor University, Deiniol Road, Bangor, Gwynedd, LL57 2UW, UK

(2) Trace Wildlife Forensics Network, Royal Zoological Society of Scotland, Corstophine Road, Edinburgh, EH12 6TS, UK

(3) Wildlife DNA Services, Tepnel Research Products \& Services, Appleton Place, Livingston, EH54 7EZ, UK

(4) Leibniz Institute for Zoo and Wildlife Research, Alfred-Kowalke-Straße 17, 10315 Berlin, Germany

(5) Veterinary Genetics Laboratory, Faculty of Veterinary Science, University of Pretoria, Onderstepoort, South Africa

\begin{abstract}
We report the development of a fast and reliable PCR-based method for sex identification of African Rhinoceros (Ceratotherium simum and Diceros bicornis) that could easily be incorporated into fluorescent short tandem repeat (STR) profiling. A single primer pair, consisting of a fluorescently labelled forward primer and an unlabelled reverse primer, is used to co-amplify homologous fragments of a zinc finger (ZF) protein intron which exhibits size polymorphism between the $X$ and $Y$ chromosomes. In both species, the amplified ZFX and ZFY amplicons differ in size by 7 bp and can thus be differentiated by capillary electrophoresis. Blood, tissue, horn, and faecal samples were correctly sexed using this method. Cross species testing also demonstrated that this method could be used to sex Indian rhinoceros (Rhinoceros unicornis) samples.
\end{abstract}

Keywords Molecular sex identification - Ceratotherium simum - Diceros bicornis - Zinc finger protein

\section{Introduction}

Identifying the sex of individuals is an important component of species monitoring programmes (Hill et al. 2005) which can improve our understanding of population structure and dynamics, and assist management decisions. Knowledge of the sex ratio within a population is particularly important for species or populations of conservation concern, as sub-optimal sex ratios can have a negative impact on population growth and resilience (Engen et al. 2003). Sex identification is hampered in many species by a lack of sexual dimorphism, particularly in juveniles, and further complicated in rare or elusive species due to a lack of opportunities for direct observation. Several studies have developed molecular methods of sex identification for endangered species, and demonstrated the use of these techniques on non-invasive sample types (e.g. Taberlet et al. 1993; Villesen and Fredsted 2006; Durnin et al. 2007; Stratham et al. 2007). 
Rhinoceros horn is highly sought after, primarily as an ingredient in traditional Asian medicine (TAM) and as a raw material for making ornamental items. The demand for horn was the principle cause for the decline in rhinoceros numbers during the 20th century, and despite international legislation and domestic bans on rhinoceros horn trade, poaching and illegal activity continue to threaten the world's rhinoceros species today (Amin et al. 2006). The two species of African rhinoceros - the white rhinoceros Ceratotherium simum and the black rhinoceros Diceros bicornis - are listed as 'near threatened' and 'critically endangered', respectively, on the IUCN Red List of Threatened Species (IUCN 2008). Population monitoring of these species is therefore vital to secure long-term survival, particularly as poaching can alter the sex structure of a population (Berger and Cunningham 1995).

Although the gender of adult rhinoceroses can be determined by an experienced observer, the observer's view may be hampered by distance, position relative to the animal or vegetation. Furthermore, juvenile rhinos are difficult to sex, and the carcasses of poached individuals have often decomposed to the extent that gender assignment is impossible.

Here, we report the development of a non-invasive molecular sexing technique for African rhinoceros species. In mammals, two main approaches are utilised for molecular sex identification, namely (a) amplification of the Y chromosome specific SRY gene (e.g. Dallas et al. 2000) and (b) amplification of homologous fragments on the $\mathrm{X}$ and $\mathrm{Y}$ chromosomes, such as the amelogenin gene (AMELX and AMELY) or zinc finger protein genes (ZFX and ZFY) (e.g. Xu et al. 2007). Tests based on SRY amplification can be problematic as nonamplification of the Y marker represents either a female sample or a failed PCR, so additional PCR controls must be designed and implemented. Tests based on amplification of homologous fragments on the $\mathrm{X}$ and $\mathrm{Y}$ chromosomes utilise either size polymorphism, usually elucidated by electrophoresis on agarose gels, or the presence of a Y-specific restriction site for RFLP analysis. However, for both these approaches, PCR products of considerable length are required, making them unsuitable for the analysis of degraded DNA (e.g. faecal samples). In this study, we exploit a small size difference (approx. $7 \mathrm{bp}$ ) between ZFX and ZFY homologs — elucidated by capillary electrophoresis - to determine the sex of African rhino samples, and demonstrate it's effectiveness on diverse sample types. The current research forms part of a larger project to develop markers for conservation genetic studies and for linking horns to their individual source animal, in order to help investigate illegal poaching and trade of African rhinoceros. 


\section{Methods}

\section{Samples}

Various sample types were obtained from wild and captive C. simum and D. bicornis of known sex. C. simum samples: blood (female $=10$, male $=10)$, tissue $(f=2, m=3)$, horn $(f=10, m=10)$, faeces $(f=1, m=1)$. D. bicornis: blood $(f=2, m=2)$, tissue $(m=1)$, horn $(f=2, m=2)$. R. unicornis samples were also obtained: blood $(f=1)$, horn $(m=1)$.

\section{DNA extraction}

DNA was extracted from blood and tissue samples using an Invitrogen ${ }^{\mathrm{TM}}$ PureLink $^{\mathrm{TM}}$ Genomic DNA Mini Kit following the manufacturer's instructions. DNA was extracted from the horn samples following the 'tissue' protocol, except that dithiothreitol (DTT, $40 \mathrm{mM}$ ) was added to the digestion buffer during the lysate preparation stage, and the total incubation time was $12 \mathrm{~h}$ (an additional $40 \mu \mathrm{l}$ proteinase $\mathrm{K}$ was added after $6 \mathrm{~h}$ ). DNA was extracted from frozen faecal samples using the QIAamp DNA Stool Mini Kit (QIAGEN ${ }^{\circledR}$ ) following the manufacturers recommendations. Extraction controls were run in parallel to all extractions. Total extracted DNA was quantified by absorbance using a NanoDrop ${ }^{\circledR}$ ND-1000 UV-Vis Spectrophotometer (Labtech International, UK).

\section{PCR amplification}

A single primer pair was designed on a consensus sequence generated from ZFX and ZFY sequences from C. simum and D. bicornis obtained from GenBank (see Table 1). The fluorescently labelled forward primer ARZF1_f (5'-FAM-

GATTTGGAASCTAGGCATTTCC-3') and the unlabelled reverse primer ARZF1_r (5'GCCATGATACTCATGAATGACA-3') target a region which exhibits a 7 bp difference between the $\mathrm{X}$ and $\mathrm{Y}$ homologs. PCR mixtures (total volume $20 \mu \mathrm{l}$ ) contained $1 \times$ ThermoStart $^{\circledR}$ Reaction Buffer, 0.5 units Thermo-Start Taq DNA Polymerase, $2.5 \mathrm{mM} \mathrm{MgCl}$, $1.5 \mu \mathrm{M}$ of each primer and 2-20 ng of template DNA. Amplifications were carried out using a PTC-200 MF Research thermocycler with the following conditions: initial denaturation at $95^{\circ}$ for 45 ; followed by 32 cycles of denaturation at $95^{\circ} \mathrm{C}$ for $45 \mathrm{~s}$, annealing at $55^{\circ} \mathrm{C}$ for $20 \mathrm{~s}$, and extension at $72^{\circ} \mathrm{C}$ for $30 \mathrm{~s}$; followed by a final extension step at $72^{\circ} \mathrm{C}$ for $10 \mathrm{~min}$. For the horn and faecal DNA extracts, the number of cycles was increased to 40. All amplifications included a negative control without template DNA, and a positive control containing $10 \mathrm{ng}$ of 
C. simum DNA. Amplification products were visualised under UV using ethidium bromide stained agarose gels, then diluted 10-fold in ABI Hi-Di Formamide before capillary electrophoresis on an Applied Biosystems Inc. 3130xl Genetic Analyzer (ABI). Alleles were sized against an internal standard Genescan ${ }^{\mathrm{TM}} 500 \mathrm{LIZ}^{\circledR}$ and scored using GeneMapper software v4.0.

\section{Results and discussion}

Female C. simum and D. bicornis individuals were characterised by two identical sized X chromosome amplicons $95 \mathrm{bp}$ in length. Males were characterised by the $95 \mathrm{bp} \mathrm{X}$ chromosome amplicon, and an additional Y chromosome amplicon 102 bp in length, making them easily distinguishable from females (see Fig. 1). Furthermore, the same amplicons were obtained from the Indian rhinoceros samples ( $\mathrm{f}=95 \mathrm{bp}, \mathrm{m}=95 \mathrm{bp}, 102 \mathrm{bp}$ ), suggesting that this technique may also be applied to Asian rhino species, although further testing is required.
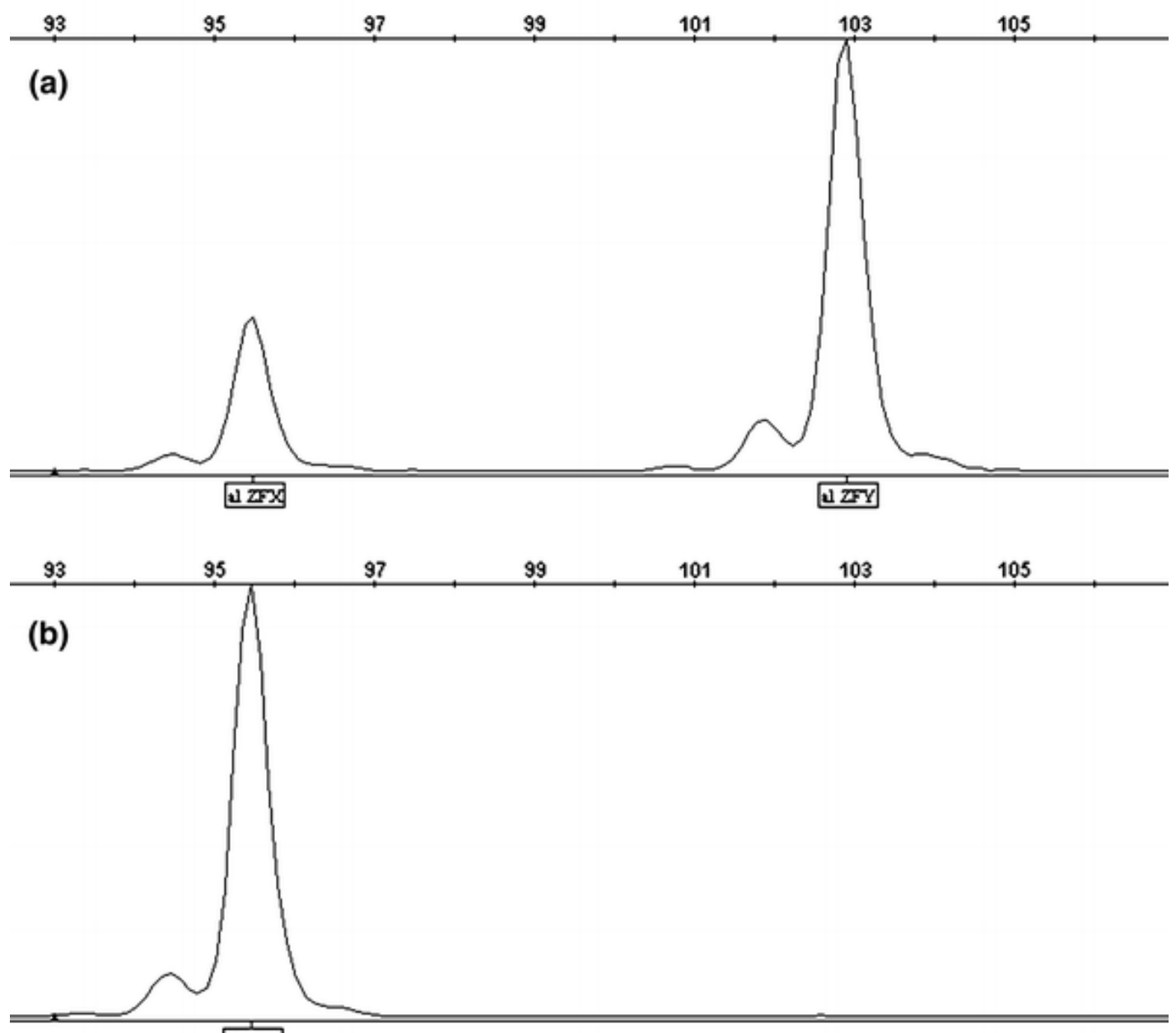

\section{나잦}

Fig. 1 Electropherogram image showing results produced by a male and $\boldsymbol{b}$ female samples 
Table 1 Table detailing the region amplified by the primers ARZF1_f and ARZF1_r

\begin{tabular}{|c|c|c|c|c|c|c|c|c|c|c|c|c|c|c|c|c|c|c|c|c|c|c|c|c|c|c|}
\hline \multirow{2}{*}{$\begin{array}{l}\text { Species / Gene } \\
\text { C. simum, ZFX }\end{array}$} & \multicolumn{19}{|c|}{ Accession No. } & \multicolumn{7}{|c|}{ Nucleotide } \\
\hline & \multicolumn{5}{|c|}{ DQ519375 } & G & A & $\mathbf{T}$ & $\mathbf{T}$ & $\mathrm{T}$ & G & G & A & A $G$ & $\mathrm{C}$ & $\mathrm{T}$ & A & G C & G $\quad \mathrm{C}$ & $\mathrm{CA}$ & $\mathrm{T}$ & $\mathrm{T}$ & $T$ & c c & \multicolumn{2}{|c|}{ C T } \\
\hline D. bicornis, ZFX & & DQ: & 193 & 374 & & . & . & . & . & . & . & . & . & . . & . . & . & . & . & . . & . . & . & . & . & . & . & . \\
\hline C. simum, ZFY & & DQ: & 206 & 345 & & . & . & . & . & . & . & . & . & . $\mathrm{c}$ & . & . & . & . . & . . & . . & . & . & . & . & . & . \\
\hline D. bicornis, ZFY & & DQ: & 206 & 644 & & . & . & . & . & . & . & . & . & . c & $=$ & . & . & . . & . . & . & . & . & . & . & . & . \\
\hline \multicolumn{27}{|l|}{ Species / Gene } \\
\hline C. simum, ZFX & $\mathrm{T}$ & A & G & $\mathrm{T}$ & A & A & A & $\mathrm{T}$ & - & - & - & - & - & G $T$ & T - & - & - & - & $-c$ & A & $\mathrm{T}$ & $\mathrm{A}$ & A & A & A & $\mathrm{c}$ \\
\hline D. bicornis, ZFX & . & . & A & . & . & . & $\cdot$ & . & . & . & . & . & . & . . & . . & . & . & . & . . & . & . & . & . & . & . & . \\
\hline C. simum, ZFY & . & . & - & . & . & . & . & . & A & $T$ & A & G & $\mathrm{T}$ & $G \quad T$ & T A & $\mathbf{T}$ & G & c & c c & . & . & . & . & . & G & . \\
\hline D. bicornis, ZFY & . & . & - & . & . & . & . & . & A & $\mathrm{T}$ & A & $G$ & $\mathrm{~T}$ & G T & I A & $\mathrm{T}$ & $G$ & c c & $c c$ & . & . & . & . & . & G & . \\
\hline \multicolumn{27}{|l|}{ Species / Gene } \\
\hline C. simum, ZFX & A & A & T & G & G & G & G & A & $\mathbf{A}$ & $\mathrm{T}$ & $T$ & $T$ & c & T $\quad G$ & G & c & A & T & T C & A & $\mathrm{T}$ & $\mathrm{G}$ & $\mathrm{A}$ & G & $\mathrm{T}$ & A \\
\hline D. bicornis, ZFX & . & $\cdot$ & . & . & $\cdot$ & - & $\cdot$ & . & . & $\cdot$ & $\cdot$ & . & . & . & . . & $\cdot$ & · & . & . . & . & . & . & . & · & · & $\cdot$ \\
\hline C. simum, ZFY & . & - & - & A & - & . & . & . & . & . & . & . & . & . & . & $\cdot$ & . & . & . & . & $\cdot$ & . & . & . & . & . \\
\hline \multirow[t]{2}{*}{ D. bicornis, ZFY } & $\cdot$ & $\cdot$ & . & A & $\cdot$ & $\cdot$ & $\cdot$ & . & . & . & . & . & . & . & . & . & . & $\cdot$ & . . & . & $\cdot$ & . & . & . & . & . \\
\hline & & & & & & & & & & & & & & & & & & & & & & Rev & vers & se $P$ & 'rime & \\
\hline
\end{tabular}

The region amplified relates to bp 353-461 of a consensus sequence generated by aligning all four sequences. The first base of the forward primer corresponds to base position 272 of GenBank sequence DQ519375

Sex was correctly assigned for all sample types, including faecal and horn extracts, confirming that this method is applicable to non-invasive monitoring (e.g. faecal analysis). In addition to assessing the sex structure of rhinoceros populations, the technique could be combined with other molecular markers (such as microsatellites) for more detailed population analyses. Furthermore, sex identification alleles can be very powerful forensic markers for matching trace evidence to individuals, as their roughly equal population frequency allows $50 \%$ of potential source animals to be immediately excluded. Work is ongoing in our laboratories to incorporate the sex identification test into a forensic STR-based assay for the analysis and protection of rhinoceros (Peppin et al. in preparation).

Acknowledgments The authors are very grateful to the organisations and individuals which provided samples for this study: The Veterinary Genetics Laboratory, Onderstepoort Faculty of Veterinary Science, Berlin Zoo, Budapest Zoo, Munich Zoo, Osnabrück Zoo, Bratislava Zoo, Selwo Adventure Park, West Midland Safari Park, Paris Zoo, Münster Zoo, Ústí Zoo, Dublin Zoo and Salzburg Zoo. This project was part funded by the Objective 1-European Social Fund. 


\section{References}

Amin R, Thomas K, Emslie RH, Foose TJ, Van Strien N (2006) An overview of the conservation status of and threats to rhinoceros species in the wild. Int Zoo Yearb 40:96-117.

Berger JB, Cunningham C (1995) Predation, sensitivity, and sex: why female black rhinoceroses outlive males. Behav Ecol 6:57-64.

Dallas JF, Carss DN, Marshall F, Koepfli K-P, Kruuk H, Piertney SB, Bacon PJ (2000) Sex identification of the Eurasia otter Lutra lutra by PCR typing of spraints. Conserv Genet $1: 181-183$.

Durnin ME, Palsbøll PJ, Ryder OA, McCullough DR (2007) A reliable genetic technique for sex determination of giant panda (Ailuropoda melanoleuca) from non-invasively collected hair samples. Conserv Genet 8:715-720.

Engen S, Lande R, Saether BE (2003) Demographic stochasticity and Allee effects in populations with two sexes. Ecology 84(9):2378-2386.

Hill DA, Fasham M, Tucker G, Shrewry M, Shaw P (2005) Handbook of biodiversity methods: survey, evaluation and monitoring. Cambridge University Press, Cambridge

IUCN (2008) IUCN Red list of threatened species, http://www.iucnredlist.org/. Downloaded on 10 November 2008

Stratham MJ, Turner PD, Reilly CO (2007) Molecular sex identification of five mustelid species. Zool Stud 46(5):600-608

Taberlet P, Mattock H, Dubois-Paganon C, Bouvet J (1993) Sexing free-ranging brown bears Ursus arctos using hairs found in the field. Mol Ecol 2:399-403.

Villesen P, Fredsted T (2006) A new sex identification tool: one primer pair can reliably sex ape and monkey DNA samples. Conserv Genet 7:455-459.

Xu X, Lin L, Zhang Z, Shen F, Zhang L, Yue B (2007) A reliable, non-invasive PCR method for giant panda (Ailuropoda melanoleuca) sex identification. Conserv Genet 9:739-741. 